\title{
SYK model, chaos and conserved charge
}

\section{Ritabrata Bhattacharya, ${ }^{a}$ Subhroneel Chakrabarti, ${ }^{a}$ Dileep P. Jatkar ${ }^{a}$ and Arnab Kundu ${ }^{b}$}

${ }^{a}$ Harish-Chandra Research Institute (HBNI), Chhatnag Road, Jhusi, Allahabad 211019, India

${ }^{b}$ Theory Division, Saha Institute of Nuclear Physics (HBNI), 1/AF Bidhannagar, Kolkata 700064, India

E-mail: ritabratabhattacharya@hri.res.in, subhroneelchak@hri.res.in, dileep@hri.res.in, arnab.kundu@saha.ac.in

ABSTRACT: We study the SYK model with complex fermions, in the presence of an all-toall $q$-body interaction, with a non-vanishing chemical potential. We find that, in the large $q$ limit, this model can be solved exactly and the corresponding Lyapunov exponent can be obtained semi-analytically. The resulting Lyapunov exponent is a sensitive function of the chemical potential $\mu$. Even when the coupling $J$, which corresponds to the disorder averaged values of the all to all fermion interaction, is large, values of $\mu$ which are exponentially small compared to $J$ lead to suppression of the Lyapunov exponent.

Keywords: 1/N Expansion, Field Theories in Lower Dimensions, Renormalization Group ArXiv EPrint: 1709.07613 


\section{Contents}

1 Introduction 1

2 SYK model with complex fermions and chemical potential 3

2.1 The SYK model 3

2.2 SYK model with complex fermions 4

2.3 Free fermion propagator, with a chemical potential 4

2.4 Differential equation for $g(\mu, \tau) \quad 5$

$\begin{array}{lll}3 & \text { Calculating the retarded kernel } & 7\end{array}$

$\begin{array}{ll}3.1 \text { The retarded Green's function } & 7\end{array}$

$\begin{array}{lll}3.2 & \text { The retarded kernel } & 7\end{array}$

$\begin{array}{lll}4 & \text { Exploring the chaos regime } & 9\end{array}$

5 Flavoured complex fermions with a chemical potential $\quad 11$

$\begin{array}{lll}6 & \text { Conclusion } & 13\end{array}$

\section{Introduction}

Given a quantum dynamical system, e.g., a specific Hamiltonian, a ubiquitous feature is the chaotic property of the same, which subsequently leads to ergodicity, thermalization and similar universal and coarse-grained description [1]. For classical dynamical systems, the measure of chaos is simple: a response of the classical trajectories with respect to initial conditions [1]. Quantum mechanically, although may not be unique, a quantitative measure can be given in terms of the square of commutators of self-adjoint operators that are time-separated. Specifically, from the large time behaviour of the same which typically takes the form of an exponential growth in time, one can extract the quantum analogue of the Lyapunov exponent, that, for classical dynamical systems, measures the sensitivity of two initially nearby (in the space of initial conditions) trajectories with respect to the corresponding initial conditions, as time evolves to large values.

The definition of the Lyapunov exponent, and equivalently the quantitative notion of quantum chaos, is naturally associated with large time limit of a dynamical system and therefore can be interpreted as an inherently infra-red (IR) quantity. Within the purview of quantum field theory (QFT) a la Wilson, one begins with an ultra-violet (UV) description of a system, subsequently integrates out the massive modes and arrives at an effective IR description. Given a QFT at the UV, the corresponding Lyapunov exponent can be extracted from the large time behaviour of out-of-time-ordered (OTO) correlation function [2]. The resulting Lyapunov exponent is a non-trivial function of the dimensionless 
couplings that define the UV-theory. In principle, a renormalization group (RG) flow maps the set of UV couplings to a set of IR-couplings, and thus the Lyapunov exponent is a different non-trivial function of the IR-couplings [3].

In general, given a QFT or a quantum mechanical system, it is non-trivial to obtain the Lyapunov exponent. Recently, a lot of progress has been made in the Sachdev-Ye-Kitaev (SYK) model [4-6], in which the Lyapunov exponent has been analytically calculated (see e.g. [7]) and subsequently demonstrated to satisfy the maximal chaos bound, proposed and argued in [8]. Motivated by this, specially the saturation of the maximal bound which is thought to be a necessary condition for a quantum system to have a holographic description, connections of $\mathrm{AdS}_{2} / \mathrm{CFT}_{1}$ have been explored further, beginning with [9] and followed up by a large volume of work on the SYK model and its various generalisations, involving complex fermions, tensor models and higher dimensional analogs [10-28]. Supersymmetric generalisations of the SYK model have also been studied [18, 29, 30]. The proposed holographic dual in terms of the Schwarzian action has also been analysed [31].

Although the precise connection in terms of AdS/CFT remains unclear, the SYK model is undoubtedly unique in capturing the following features, all at once: solvable at large $N$, emergence of conformal invariance in the IR and maximal chaos. Emergence of $\mathrm{AdS}_{2}$, on the other hand, is rather unique in stringy physics: ranging from the entropy counting of extremal black hole horizons to the emergent IR description of a large $N$ strongly coupled gauge theory with non-vanishing density. Thus, a physical result obtained from the SYK model is likely to be relevant about the physics of $\mathrm{AdS}_{2}$, viewed in the appropriate context.

In this article, we explore a simple way to tune chaos in the SYK-type model, by introducing global conserved charges. We focus on the SYK-model with complex fermions, that have previously been studied in e.g. [32, 33], where the fermions have an all-to-all $q$-body interaction, with Gaussian random distribution for the coupling strength. The standard SYK model corresponds to taking $q=4$, however, similar to [33], we study the limit of $q \rightarrow \infty$. This limit particularly facilitates analytical calculations, where much of the large $q$ analysis of [7] can be generalized in the presence of a non-vanishing global charge, to obtain the corresponding Lyapunov exponent. There is another method of getting tunable Lyapunov exponent by coupling peripheral fermions to the SYK model [34].

In this case, the UV-theory comes equipped with two independent couplings: $\{\beta J, \beta \mu\}$, where $\beta=T^{-1}$ is the inverse temperature, $J$ measures the interaction strength after performing a random averaging, and $\mu$ is the chemical potential corresponding to the global charge, which introduces a new scale in the problem. In the large $N$, large $q$ limit, with $N \geq q$, the resulting Schwinger-Dyson equation regroups the UV couplings to an effective IR coupling, such that the RG flow maps $\{\beta J, \beta \mu\} \rightarrow \beta \tilde{J}$. The large $q$ analysis now yields the Lyapunov exponent: $\lambda_{\mathrm{L}}(\beta \mu, \beta J) \equiv \lambda_{\mathrm{L}}(\beta \tilde{J})$. It turns out that, by tuning the UV data one can smoothly interpolate between $\lambda_{\mathrm{L}}=2 \pi T$ to $\lambda_{\mathrm{L}}=0$. We also obtain a similar result for the complex fermions with a global flavour symmetry, introduced in [12]. The non-invertible map of the couplings, from UV to IR, emerges at large $q$, even when sub-leading effects in $(1 / q)$ are considered, and may be an artefact of this limit. ${ }^{1}$

\footnotetext{
${ }^{1}$ This is an interesting issue to explore further. It may happen that an attractor type behaviour exists, in which the deep IR physics reorganizes itself in terms of emerging parameters, irrespective of the value of $q$.
} 
That the presence of global charges suppress the Lyapunov exponent and, in fact, can tune it to vanishing values is no surprise. Conserved charges constrain the phase-space of any dynamical system, the extreme limit of which are represented by integrable models. For the latter, no chaotic behaviour is expected. Thus, the result above interpolates between a chaotic behaviour to a non-chaotic regime, even with a $\mathrm{U}(1)$ charge, as the corresponding chemical potential is increased. Similar feature upholds for the flavoured complex fermion models.

In terms of the Schwinger-Dyson equation, at large $N$, exploring the strong coupling phase of the system is equivalent to taking a deep IR limit. Keeping the effect of a nonvanishing chemical potential is similar to working at an intermediate energy-scale. In fact, Schwinger-Dyson equation comes equipped with a term $(i \omega+\mu)$, where $\omega$ is the frequency, and thus $\mu$ and $\omega$ seem freely tradable. From the UV-perspective, the interpretation is physically distinct, but for an intermediate-scale observer, studying $\lambda_{\mathrm{L}}$ as a function of $\beta \mu$ is similar to studying how $\lambda_{\mathrm{L}}$ changes away from the IR-conformal limit. ${ }^{2}$ The dependence of $\lambda_{\mathrm{L}}$ with $\beta J$ have already been explored in [7], in the $q \rightarrow \infty$ limit.

This article is divided in the following sections: in section 2 , we briefly introduce the model with complex fermions, obtain the Schwinger-Dyson equation and present the solution in the $q \rightarrow \infty$ limit. We subsequently discuss the calculation of the retarded kernel in the next section. Section 4 is devoted to studying the dependence of the Lyapunov exponent, in details. We comment briefly on flavoured complex fermion model in section 5. Finally, we conclude with future directions.

\section{SYK model with complex fermions and chemical potential}

\subsection{The SYK model}

We will begin by briefly recalling the SYK model. The SYK model describes all-to-all random interactions between $N$ Majorana fermions in $(0+1)$ dimension involving $q$ fermions at a time. The Hamiltonian is given by $[5-7]$

$$
H=(i)^{q / 2} \sum_{1 \leq i_{1} \leq \ldots . . i_{q} \leq N} j_{i_{1} \ldots i_{q}} \psi_{i_{1}} \ldots \psi_{i_{q}}
$$

where $q \leq N$ and $q=$ even. The set of couplings $\left\{j_{i_{1} \ldots i_{q}}\right\}$ are drawn from a random distribution, such as a Gaussian one, described by

$$
\mathcal{P}\left(j_{i_{1} \ldots i_{q}}\right)=\exp \left[-\frac{N^{3} j_{i_{1} \ldots i_{q}}^{2}}{12 J^{2}}\right],
$$

where $\mathcal{P}$ denotes the probability distribution. The gaussian distribution for a random variable means the average value of the couplings $j_{i_{1} \ldots i_{q}}$ is zero and the two point average with all indices contracted is non-vanishing,

$$
\left\langle j_{i_{1} \ldots i_{q}}\right\rangle=0, \quad\left\langle j_{i_{1} \ldots i_{q}}^{2}\right\rangle=\frac{J^{2}(q-1) !}{N^{q-1}} .
$$

\footnotetext{
${ }^{2}$ We note here that, even in the presence of a chemical potential, that defines a scale for the system, in the deep IR conformal symmetry is recovered, when supplemented by a gauge transformation. See e.g. [32].
} 
The Majorana condition on the fermions simply means that they satisfy the anticommutation relation,

$$
\left\{\psi_{i}, \psi_{j}\right\}=\delta_{i j}
$$

The Lagrangian corresponding to (2.1) is given by

$$
\begin{array}{rlrl}
S & =\int d \tau L_{\mathrm{E}}\left(\left\{\psi_{i}\right\},\left\{\frac{d \psi_{i}}{d \tau}\right\}\right), & L_{\mathrm{E}} & =\frac{1}{2} \psi_{i} \frac{d \psi_{i}}{d \tau}-H, \\
\text { equivalently } L & =-\frac{1}{2} \psi_{i} \frac{d \psi_{i}}{d t}-H, \quad \text { with } \quad t=-i \tau .
\end{array}
$$

In the above $L_{\mathrm{E}}$ and $L$ corresponds to the Lagrangian in Euclidean and Minkowski signatures, respectively.

\subsection{SYK model with complex fermions}

In order to introduce a chemical potential, we will explore the model involving complex fermions. This model has been studied earlier in the condensed matter context [32], focussing on transport properties and thermodynamics; and in the context of chaos in [33]. We are interested in the large $q$ expansion of the complex fermion model with an addition of a non-vanishing chemical potential, which seems analogous to adding a mass term.

The Hamiltonian for the SYK model with complex fermions is

$$
H=\sum J_{i_{1} i_{2} \ldots i_{q / 2} i_{q / 2+1} \ldots i_{q}} \psi_{i_{1}}^{\dagger} \psi_{i_{2}}^{\dagger} \ldots \psi_{i_{q / 2}}^{\dagger} \psi_{i_{q / 2+1}} \ldots \psi_{i_{q}} .
$$

In what follows we will use the notations and conventions used in [32]. In addition to this interaction term we introduce a chemical potential $\mu$. We are interested in studying the effect of a conserved charge on the chaotic behaviour of the model. Some of the earlier works [32, 33] have analysed this model with either quartic interactions or in the nonchaotic regime. We will work in the large $q$ limit and find out how the Lyapunov exponent changes as we tune in the chemical potential.

\subsection{Free fermion propagator, with a chemical potential}

We define, following [32], the Green's function to be: $G(\tau)=-\left\langle\mathrm{T} \psi(\tau) \psi^{\dagger}(0)\right\rangle$, where the symbol $\mathrm{T}$ stands for time-ordering and $\tau$ is the imaginary time. The free fermion propagator, in the Fourier space, takes the form:

$$
G(\mu, \omega)=\frac{1}{i \omega+\mu},
$$

which, in the real space, corresponds to the operator $\left(-\partial_{t}+\mu\right)$. The two point function in the interacting theory, in the large $q$ limit, can be expanded as:

$$
G(\mu, \tau)=G_{0}(\mu, \tau)\left(1+\frac{g(\mu, \tau)}{q}+\ldots\right)
$$

where $G_{0}(\mu, \tau)$ is the Fourier transform of the free propagator, which at zero temperature it is given by,

$$
G_{0}(\mu, \tau)=-e^{\mu \tau} \Theta(-\tau)
$$


Here $\Theta$ is the Heaviside step function. At non-vanishing temperature, however, it is obtained by evaluating the sum over Matsubara frequencies that appear in the propagator, $\left(i \omega_{n}+\mu\right)^{-1}$, which yields,

$$
\begin{aligned}
G_{0}(\mu, \tau) & =-\frac{e^{\mu \tau}}{e^{\mu \beta}+1}, & 0 & \leq \tau \leq \beta, \\
G_{0}(\mu, \tau) & =\frac{e^{\mu \tau}}{e^{-\mu \beta}+1}, & -\beta & \leq \tau \leq 0 .
\end{aligned}
$$

The propagator for $\tau<0$ is obtained using the periodicity $\tau \rightarrow \tau+\beta$. The relative sign between $\tau<0$ and $\tau>0$ is a reflection of the fact that $G_{0}(\mu, \tau)$ is a fermion propagator. Finally, the function $g(\mu, \tau)$ is the correction due to melonic diagrams to the free propagator, in the large $q$ limit. In the next subsection we will derive a differential equation for $g(\mu, \tau)$ and subsequently solve it.

\subsection{Differential equation for $g(\mu, \tau)$}

To derive the desired differential equation, we follow a simple generalisation of the method discussed in [7]. First, note that, in the large $N$ limit, all melonic Feynman diagram can be summed up to obtain the following Schwinger-Dyson equation:

$$
\begin{aligned}
\frac{1}{G(\mu, \omega)} & =i \omega+\mu-\Sigma(\omega, \mu), \\
\Sigma(\omega, \mu) & =J^{2}(-1)^{q / 2}(G(\mu, \tau))^{q / 2}(G(\mu,-\tau))^{q / 2-1} .
\end{aligned}
$$

It is straightforward to derive the above Schwinger-Dyson equations by summing up the one particle irreducible diagrams. Specifically, it is straightforward to observe the second line above via Feynman diagrammatic, see figure 1.

These Schwinger-Dyson equations take especially simple form in the $q \rightarrow \infty$ limit. In particular, the function $g(\mu, \tau)$ in this limit appears in the exponential:

$$
\begin{aligned}
\frac{1}{G(\mu, \omega)} & =i \omega+\mu-(i \omega+\mu)^{2} \frac{f * g(\mu, \omega)}{2 q} . \\
\Sigma(\mu, \tau) & =\frac{J^{2} G_{0}(\mu, \tau)}{(2+2 \cosh (\mu \beta))^{q / 2-1}} e^{\frac{1}{2}(g(\mu, \tau)+g(\mu,-\tau))} .
\end{aligned}
$$

We can now identify the self energy contribution to the inverse propagator as the Fourier transform of $\Sigma(\mu, \tau)$ appearing in (2.16). Taking the inverse Fourier transform of the self energy contribution in (2.15) we get the differential equation:

$$
\left(\partial_{t}-\mu\right)^{2}\left[G_{0}(\mu, \tau) g(\mu, \tau)\right]=2 \frac{q J^{2} G_{0}(\mu, \tau)}{2(2+2 \cosh (\mu \beta))^{q / 2-1}} e^{\frac{1}{2}(g(\mu, \tau)+g(\mu,-\tau))} .
$$

For $\tau>0$ this equation reduces to:

$$
\partial_{\tau}^{2} g(\mu, \tau)=2 \tilde{J}^{2} e^{\frac{1}{2}(g(\mu, \tau)+g(\mu,-\tau))},
$$

where,

$$
\tilde{J}^{2}=\frac{q J^{2}}{2(2+2 \cosh (\mu \beta))^{\frac{q}{2}-1}}
$$




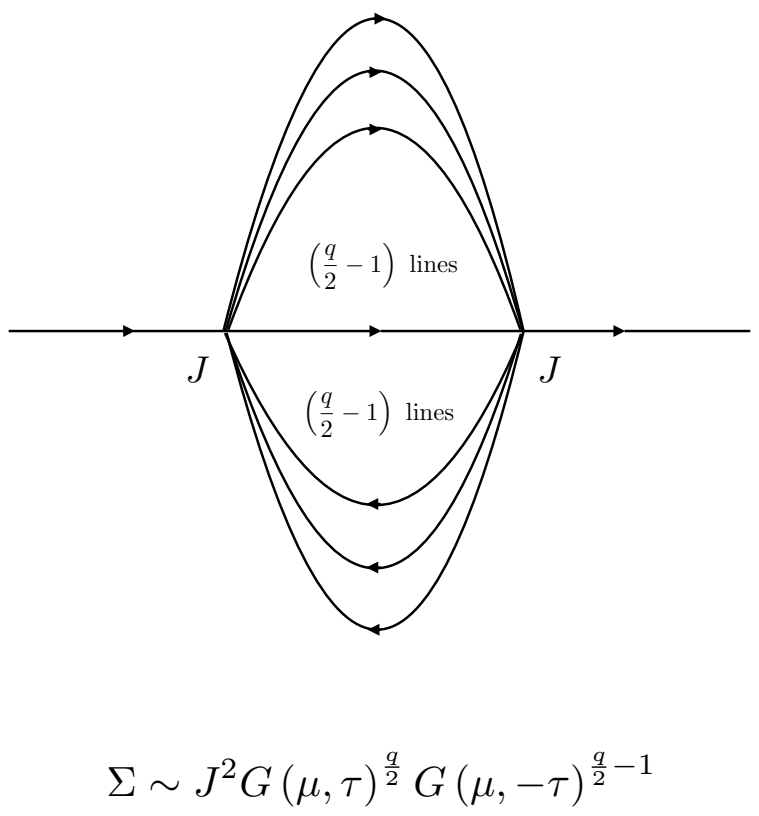

Figure 1. A diagrammatic representation of $\Sigma$. Each vertex is worth of strength $J$, and $\left(\frac{q}{2}-1\right)$ propagators run inside the loop in each direction. The direction of the arrows correlate with the sign of $\tau$ in the argument of the propagators. The overall direction of the diagram, from left to right, selects out two additional propagators running in this direction and hence the corresponding powers of $G$.

It is worth pointing out at this point that this differential equation is quite similar to that appearing in [7]. We will solve this equation analytically in the next section.

Before moving further, a few comments regarding the large $q$ result are in order. It is straightforward to check that, if one goes beyond the leading order in $(1 / q)$-expansion, the Schwinger-Dyson equation again rearranges itself to the differential equation of the type discussed above, with the same effective coupling $\tilde{J}$.

To see this explicitly let us first notice that the $\mu$ dependence of $\tilde{J}$ comes only from the free part. If we look at the behavior of the self-energy contribution at $O\left(\frac{1}{q^{2}}\right)$ we find for $\mu=0$ case, the terms take the form

$$
J^{2}\left(1+\frac{g(\tau)}{q}+\frac{g^{\prime}(\tau)}{q^{2}}+\ldots\right)^{q-1} .
$$

The equation for function $g^{\prime}$ cannot be obtained by simply exponentiating it, as was done for the leading correction, namely $g(\tau)$. We instead have an asymptotic series expansion in $\frac{1}{q}$. Now if we turn on finite $\mu$ then from the self-energy expression we get,

$$
\frac{J^{2}}{2(2+2 \cosh (\mu \beta))^{q / 2-1}}\left(1+\frac{g(\mu, \tau)}{q}+\frac{g^{\prime}(\mu, \tau)}{q^{2}}+\ldots\right)^{\frac{q}{2}}\left(1+\frac{g(\mu,-\tau)}{q}+\frac{g^{\prime}(\mu,-\tau)}{q^{2}}+\ldots\right)^{\frac{q}{2}-1}
$$

The form is exactly like in the SYK model. As a result the equation that we would obtain in this case will be identical to that for $g^{\prime}$ in the SYK model. In other words even for finite $\mu$, 
the effective coupling constant $\tilde{J}$ remains unaltered even at higher order in $1 / q$. The emergence of one effective coupling is an inherent feature of this asymptotic expansion in $(1 / q)$.

\section{Calculating the retarded kernel}

The right hand side of the differential equation (2.18) is symmetric under $\tau \rightarrow-\tau$, whereas on the left hand side we switch from $g(\mu, \tau) \rightarrow g(\mu,-\tau)$. We can therefore send $\tau \rightarrow$ $-\tau$, and subsequently obtain the resulting equation for $g(\mu,-\tau)$. The solutions to the differential equations are exactly of the Maldacena-Stanford form [7], and are given by

$$
e^{g(\mu, \pm \tau)}=\frac{\cos ^{2}\left(\frac{\pi \nu}{2}\right)}{\cos ^{2}\left(\pi \nu\left(\frac{\tau}{\beta} \mp \frac{1}{2}\right)\right)}, \quad \text { with } \quad \beta \tilde{J}=\frac{\pi \nu}{\cos \left(\frac{\pi \nu}{2}\right)}
$$

Note that, the parameter $\nu$ that naturally emerges here contains information about the two independent UV-couplings: $\beta J$ and $\beta \mu$.

\subsection{The retarded Green's function}

We begin by defining the retarded Green's function

$$
G_{\mathrm{R}}(\mu, t)=\lim _{\epsilon \rightarrow 0+}\left[G_{>}(\mu, i t+\epsilon)-G_{<}(\mu, i t-\epsilon)\right] \Theta(t) .
$$

In the $q \rightarrow \infty$ limit, we obtain:

$$
G_{\mathrm{R}}(\mu, t)=-e^{i \mu t} \Theta(t) .
$$

The above result, in the limit $\mu \rightarrow 0$, yields: $G_{\mathrm{R}}(t)=\Theta(t)$ which is the expected answer. We can also define:

$$
G_{\mathrm{R}}(\mu,-t)=\lim _{\epsilon \rightarrow 0+}\left[G_{>}(\mu,-(i t+\epsilon))-G_{<}(\mu,-(i t-\epsilon))\right] \Theta(t),
$$

which implies $G_{\mathrm{R}}(\mu,-t)=e^{-i \mu t} \Theta(t)$.

\subsection{The retarded kernel}

Now we analyze the four-point function. In the large $N$ limit, the four-point function can be expanded in a series of $(1 / N)$ and, here, we will only compute the leading $(1 / N)$ contribution, in which only the ladder diagrams contribute. Since we are working with complex fermions, the only non-trivial four-point function is given by

$$
\frac{1}{N^{2}} \sum_{i, j=1}^{N}\left\langle T\left(\psi_{i}\left(t_{1}\right) \psi_{i}^{\dagger}\left(t_{2}\right) \psi_{j}^{\dagger}\left(t_{3}\right) \psi_{j}\left(t_{4}\right)\right)\right\rangle=G\left(t_{12}\right) G\left(t_{34}\right)+\frac{1}{N} \mathcal{F}\left(t_{1}, t_{2}, t_{3}, t_{4}\right)+\ldots
$$

The contribution at order $(1 / N)$ is collectively denoted by $\mathcal{F}=\sum_{n} \mathcal{F}_{n}$, where $n$ is the number of rungs in the corresponding ladder diagram. We refer to [7] for more details. The composition rule is pictorially represented in figure 2 . 


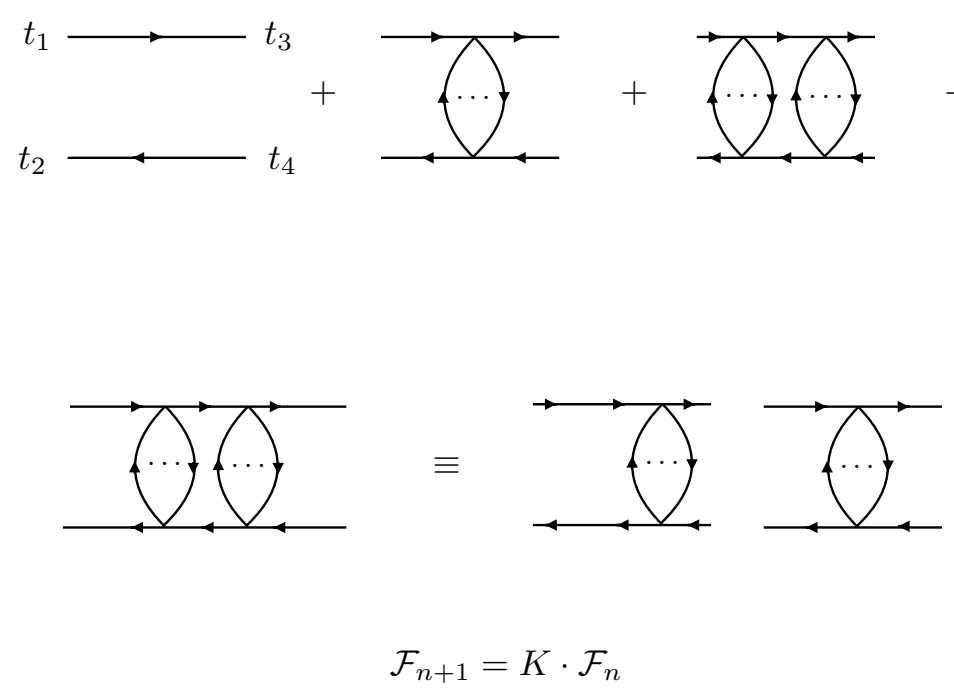

Figure 2. A diagrammatic representation of the four point function calculation, in the large $N$ limit. First, only the ladder diagrams contribute, as shown in the first row here. Second, from the structure of the diagrams, one obtains an iterative process to generate $\mathcal{F}_{n+1}$ from $\mathcal{F}_{n}$, composing with a kernel.

At large $N$, the summation over the ladder diagrams can be performed by expressing $\mathcal{F}_{n+1}$ in terms of $\mathcal{F}_{n}$ integrated, weighted with a kernel, as also pictorially shown in figure 2 :

$$
\mathcal{F}_{n+1}\left(t_{1}, t_{2}, t_{3}, t_{4}\right)=\int d t d t^{\prime} K_{\mathrm{R}}\left(t_{1}, t_{2} ; t, t^{\prime}\right) \mathcal{F}_{n}\left(t, t^{\prime}, t_{3}, t_{4}\right)
$$

where the kernel, denoted above by $K_{\mathrm{R}}$, is given by

$$
\begin{aligned}
K_{\mathrm{R}}\left(t_{1}, t_{2}, t_{3}, t_{4}\right)=(-1)^{q / 2} J^{2}(q-1) & G_{\mathrm{R}}\left(\mu, t_{13}\right) G_{\mathrm{R}}\left(\mu,-t_{24}\right) \\
& \times\left[G_{\mathrm{lr}}\left(\mu, t_{34}\right)\right]^{q / 2-1}\left[G_{\mathrm{lr}}\left(\mu,-t_{34}\right)\right]^{q / 2-1} .
\end{aligned}
$$

Here $G_{\mathrm{lr}}(\mu, t)$ is the Wightman function, which is essentially given by the propagator evaluated at complex time, and in the large $q$ limit we get:

$$
\left[G_{\mathrm{lr}}(t)\right]^{q / 2-1}\left[G_{\mathrm{lr}}(-t)\right]^{q / 2-1}=[G(i t+\beta / 2)]^{q / 2-1}[G(-i t+\beta / 2)]^{q / 2-1} .
$$

The above is consistent with interpreting the propagator $G(\mu,-t)$ as the fermion moving backward in time, or the anti-fermion moving forward in time. This is why a separation along the thermal circle picks up a relative sign.

Finally, we obtain:

$$
(-1)^{q / 2} J^{2}(q-1)\left[G_{\mathrm{lr}}(t)\right]^{q / 2-1}\left[G_{\mathrm{lr}}(-t)\right]^{q / 2-1}=(-1)^{q-1} \frac{2 \pi^{2} \nu^{2}}{\beta^{2} \cosh ^{2}\left(\frac{\pi \nu t}{\beta}\right)} .
$$

Using this, the complete retarded kernel is given by

$$
\begin{aligned}
K_{\mathrm{R}}\left(t_{1}, t_{2}, t_{3}, t_{4}\right) & =-(-1)^{q-1} e^{i \mu\left(t_{13}-t_{24}\right)} \frac{2 \pi^{2} \nu^{2} \Theta\left(t_{13}\right) \Theta\left(t_{24}\right)}{\beta^{2} \cosh ^{2}\left(\frac{\pi \nu t_{34}}{\beta}\right)} \\
& =e^{i \mu\left(t_{12}-t_{34}\right)} \frac{2 \pi^{2} \nu^{2} \Theta\left(t_{13}\right) \Theta\left(t_{24}\right)}{\beta^{2} \cosh ^{2}\left(\frac{\pi \nu t_{34}}{\beta}\right)} .
\end{aligned}
$$

The last equality follows from the fact that $q$ is even. 


\section{Exploring the chaos regime}

So far, we have obtained the retarded kernel for four fermion fields placed at four arbitrary points on the thermal circle, denoted respectively by $t_{1}, \ldots, t_{4}$. To extract the chaos behaviour, one needs to calculate the OTO correlation in real time, separating the fermions by a quarter of the thermal circle $[5,6]$. We want to compute the following OTO correlation:

$$
\mathcal{F}\left(t_{1}, t_{2}\right)=\operatorname{Tr}\left[y \psi_{i}\left(t_{1}\right) y \psi_{i}^{\dagger}(0) y \psi_{j}^{\dagger}\left(t_{2}\right) y \psi_{j}(0)\right], \quad y=\rho(\beta)^{1 / 4} .
$$

In the limit $t_{1}, t_{2} \rightarrow \infty$, the diagram with zero rung is suppressed and thus $\mathcal{F}\left(t_{1}, t_{2}\right)$ is an eigenfunction of the retarded kernel $K_{\mathrm{R}}$, with an eigenvalue one. This statement translates into an integral equation of the following form:

$$
\begin{aligned}
\mathcal{F}\left(t_{1}, t_{2}\right) & =\int_{-\infty}^{\infty} \int_{-\infty}^{\infty} d t_{3} d t_{4} K_{\mathrm{R}}\left(t_{1}, t_{2}, t_{3}, t_{4}\right) \mathcal{F}\left(t_{3}, t_{4}\right) \\
& =\int_{-\infty}^{\infty} \int_{-\infty}^{\infty} d t_{3} d t_{4} e^{i \mu\left(t_{12}-t_{34}\right)} \frac{2 \pi^{2} \nu^{2} \Theta\left(t_{13}\right) \Theta\left(t_{24}\right)}{\beta^{2} \cosh ^{2}\left(\frac{\pi \nu t_{34}}{\beta}\right)} \mathcal{F}\left(t_{3}, t_{4}\right) .
\end{aligned}
$$

Choosing an exponential-ansatz for $\mathcal{F}\left(t_{3}, t_{4}\right)$ of the form

$$
\mathcal{F}\left(t_{3}, t_{4}\right)=e^{\frac{\pi \nu}{\beta}\left(t_{3}+t_{4}\right)} \frac{e^{i \mu t_{34}}}{\cosh \left(\frac{\pi \nu t_{34}}{\beta}\right)},
$$

yields:

$$
\begin{aligned}
\mathcal{F}\left(t_{1}, t_{2}\right) & =e^{i \mu t_{12}} \int_{-\infty}^{t_{1}} \int_{-\infty}^{t_{2}} d t_{3} d t_{4} \frac{2 \pi^{2} \nu^{2} e^{\frac{\pi \nu}{\beta}\left(t_{3}+t_{4}\right)}}{\beta^{2} \cosh ^{3}\left(\frac{\pi \nu t_{34}}{\beta}\right)} \\
& =e^{\frac{\pi \nu}{\beta}\left(t_{1}+t_{2}\right)} \frac{e^{i \mu\left(t_{12}\right)}}{\cosh \left(\frac{\pi \nu t_{12}}{\beta}\right)} .
\end{aligned}
$$

This implies, following the subsequent steps outlined in [7], that the Lyapunov exponent is given by

$$
\lambda_{\mathrm{L}}=\frac{2 \pi}{\beta} \nu,
$$

where $\nu$ is given in equation (3.1). In the two extreme limits, we easily get:

$$
\begin{aligned}
& \lambda_{\mathrm{L}}=(2 \tilde{J})+\ldots, \quad \text { as } \quad \nu \rightarrow 0 \quad \Longleftrightarrow \quad \beta \tilde{J} \rightarrow 0, \\
& =\frac{2 \pi}{\beta}\left(1-\frac{2}{\beta \tilde{J}}\right), \quad \text { as } \quad \nu \rightarrow 1 \quad \Longleftrightarrow \quad \beta \tilde{J} \rightarrow \infty \text {. }
\end{aligned}
$$

In terms of the IR emergent coupling $\beta \tilde{J}$, the dependence is identical to the one observed in [7], however, in terms of the original parameters $\{\beta J, \beta \mu\}$ defining the system, there is a non-trivial dependence of the Lyapunov exponent. The figure 3, shows behaviour of $\lambda=\beta \lambda_{L} / 2 \pi$, which is the normalised Lyapunov exponent, as a function of the coupling 


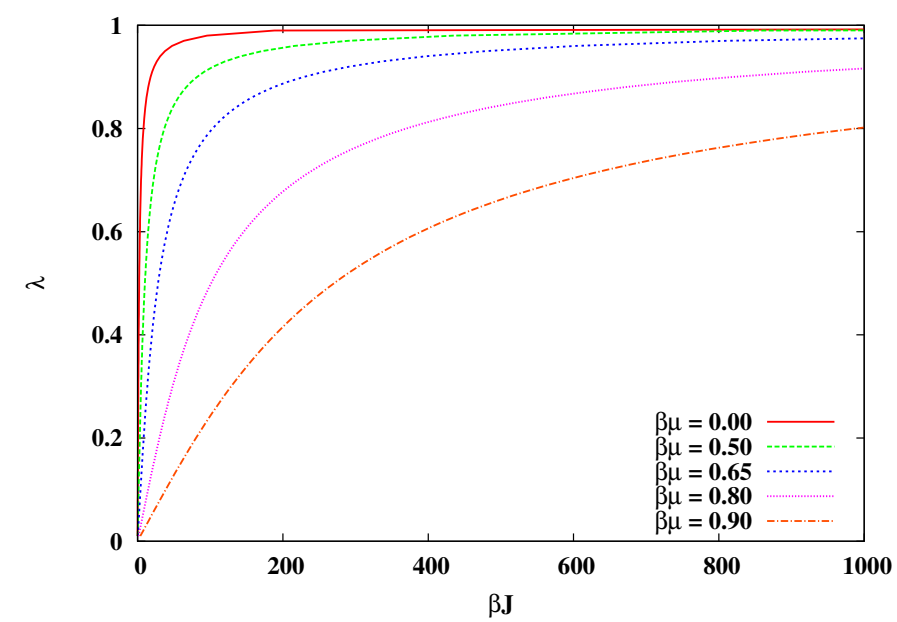

Figure 3. The Lyapunov exponent $\lambda$ is normalised and takes values between 0 and 1 . This figure shows dependence of $\lambda$ on $\beta J$ for different values of $\beta \mu$.

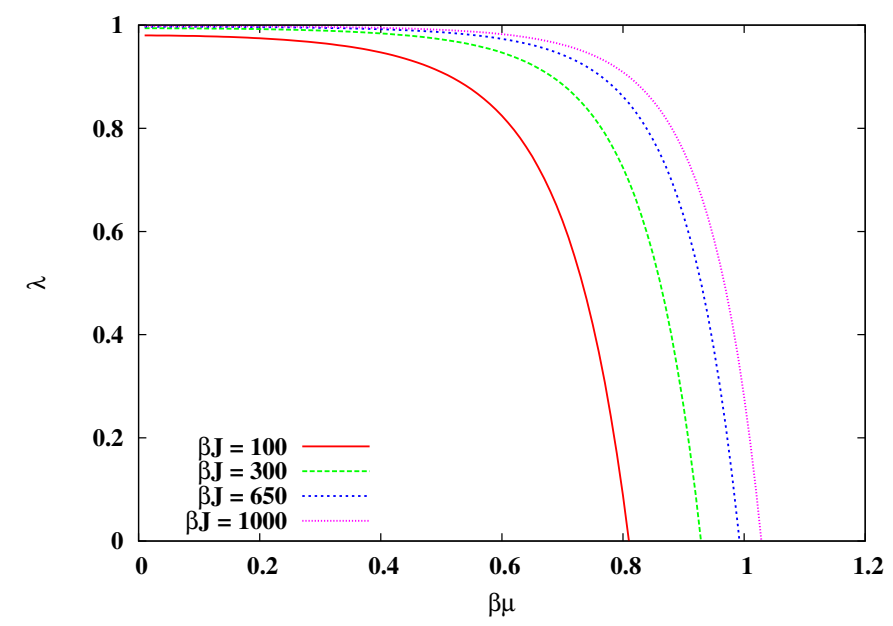

Figure 4. The Lyapunov exponent $\lambda$ is again normalised and takes values between 0 and 1 . This figure shows dependence of $\lambda$ on $\beta \mu$ for different values of $\beta J$.

$\beta J$ for various values of $\beta \mu$. Similarly the figure 4 shows variation of $\lambda$ as a function of $\beta \mu$ for different values of $\beta J$.

Before concluding this section, let us make some comments regarding tuning the chaotic properties of SYK-type models. In [15], a two-body infinite-range random interaction between Majorana fermions was introduced, in addition to the four-fermi interaction in the SYK model. It was found that this interaction can tune the Lyapunov exponent down, and in fact, push it all the way to zero, similar to what we have observed above. However, the precise dependence of the Lyapunov exponent with the one-body interaction strength is different compared to our results. 
The Hamiltonian considered in [15] is of the following form:

$$
H=\sum_{1 \leq i_{1} \leq i_{2} \leq i_{3} \leq i_{4} \leq N} J_{i_{1} i_{2} i_{3} i_{4}} \psi_{i_{1}} \psi_{i_{2}} \psi_{i_{3}} \psi_{i_{4}}+i \sum_{1 \leq i_{1} \leq i_{2} \leq N} k_{i_{1} i_{2}} \psi_{i_{1}} \psi_{i_{2}}
$$

where $J_{i_{1} i_{2} i_{3} i_{4}}$ are chosen from a familiar Gaussian ensemble, and the couplings $k_{i_{1} i_{2}}$ denote the infinite-range interaction and $\psi_{i}$ 's are Majorana fermions. Assuming $N$ is even, we can consider a particularly special case, in which $k_{i_{1} i_{2}}$ are non-random, and are characterized by a particularly nearest neighbour interaction:

$$
\begin{aligned}
k_{i_{1} i_{2}} & =k \delta_{i_{1}+1, i_{2}} \quad \text { if } \quad i_{1}=\text { odd } \\
& =0 \text { otherwise }
\end{aligned}
$$

The interaction term is now particularly simple:

$$
\begin{aligned}
H_{\mathrm{int}} & =i \sum_{i=\mathrm{odd}}^{N} k_{i, i+1} \psi_{i} \psi_{i+1} \equiv \Psi^{\dagger} \mathcal{K} \Psi, \\
\text { where } \quad \Psi^{\dagger} & =\left(\psi_{1}, \psi_{2}, \ldots \psi_{N}\right) .
\end{aligned}
$$

Evidently, the ${ }^{\dagger}$ operation is equivalent to the transpose operation since we are dealing with Majorana fermions. The matrix $\mathcal{K}$ contains the information about the nearest-neighbour interaction of (4.11). It is easy to diagonalize the coupling matrix $\mathcal{K}$, and the resulting eigenvalues are: $\left(\frac{N}{2}\right)$ copies of $\left(+\frac{k}{2}\right)$ and $\left(\frac{N}{2}\right)$ copies of $-\left(\frac{k}{2}\right)$. Suppose that $\chi_{a}^{+}$, with $a=1, \ldots, N / 2$, eigenvectors have positive eigenvalues and $\chi_{a}^{-}$, with $a=1, \ldots, N / 2$, eigenvectors have negative eigenvalues. It is also straightforward to check that: $\left(\chi^{+}\right)^{\dagger}=\chi^{-}$, thus we can drop the superscript, and subsequently the interaction term can be written as:

$$
H_{\text {int }}=k \sum_{a}^{N / 2} \chi_{a}^{\dagger} \chi_{a}, \quad \text { where } \quad\left\{\chi_{a}^{\dagger}, \chi_{b}\right\}=2 \delta_{a b} .
$$

We can now rewrite the four-body interaction in the complex $\chi$-basis. Since our starting point did not preserve the U(1)-symmetry of the complex fermion model in (2.7), the full resulting Hamiltonian does not match with the complex fermion model with $q=4$. However, in the UV, with $(J / k) \rightarrow 0$, the four-point interaction is negligible and the two systems are physically equivalent. In the IR, the two systems are completely distinct.

\section{Flavoured complex fermions with a chemical potential}

Let us now generalise this set up, where instead of a $\mathrm{U}(1)$ symmetry we have $N_{f}$ number of flavoured fermions with a global SU $\left(N_{f}\right)$ flavour symmetry, similar to the model considered in [12]. The fermions now carry two indices, $\Psi_{i}^{\alpha}$. Here the $\alpha$ is the flavour index where as $i$ is the site index. One has the following operator algebra:

$$
\left\{\Psi_{i}^{\alpha}, \Psi_{j}^{\beta}\right\}=\left\{\Psi_{i}^{\alpha \dagger}, \Psi_{j}^{\beta \dagger}\right\}=0, \quad\left\{\Psi_{i}^{\alpha}, \Psi_{j}^{\beta \dagger}\right\}=\delta_{i j} \delta^{\alpha \beta} .
$$


It is a trivial matter to find first the kinetic term without introducing the chemical potential $\mu$ it given by

$$
-\int d \tau \Psi_{i}^{\alpha \dagger} \partial_{\tau} \Psi_{i}^{\alpha}
$$

Here repeated indices are summed over unless stated otherwise.

The $\mathrm{SU}\left(N_{f}\right)$ invariant two point function in this case will be given by

$$
G(\tau)=\left\langle\Psi_{i}^{\alpha}(\tau) \Psi_{j}^{\alpha \dagger}(0)\right\rangle \equiv \frac{N_{f} \operatorname{sgn}(\tau)}{2} \delta_{i j}
$$

If we absorb this factor of $N_{f}$ into the overall normalization of the kinetic piece then we observe that now if one introduces a conserved charge $\mu$ then the relevant operator is:

$$
\frac{\mu}{N_{f}} \Psi_{i}^{\alpha} \Psi_{i}^{\alpha \dagger}
$$

We know that the interaction term should be a gauge singlet. We also require that, upon imposing reality condition on the fermions, this interaction should reduce to the corresponding interaction term in the Gross-Rosenhaus model. Under this, we intuitively write down the interaction term as:

$$
\frac{1}{N_{f}^{q / 2}} J_{i_{1} \ldots i_{q}} \Psi_{i_{1}}^{\alpha_{1} \dagger} \ldots \Psi_{i_{q / 2}}^{\alpha_{q / 2} \dagger} \Psi_{i_{q / 2}+1}^{\alpha_{q / 2}} \ldots \Psi_{i_{q}}^{\alpha_{1}} .
$$

Now we just use the melon diagrams to figure out the 1PI effective self energy contribution. Essentially, as before, we observe that from the diagramatics one obtains:

$$
\Sigma(\tau)=\frac{C_{\frac{q}{2}}^{N_{f}}}{N_{f}^{q}} J^{2}[G(\tau)]^{q / 2}[G(-\tau)]^{q / 2-1} .
$$

So, one can redefine the coupling strength as: $J_{\text {eff }}^{2}=\frac{C_{\frac{q}{2}}^{N_{f}}}{N_{f}^{q}} J^{2}$. This means that, if we have multiple groups of flavours, then the relative strength of the effective couplings scale according to the above relation. Hence, again we get back the same set of Schwinger-Dyson equations which we have already solved.

We already see the emergence of an effective coupling:

$$
J_{\text {eff }}^{2}=\frac{1}{N_{f}^{q}} \frac{N_{f} !}{\left(\frac{q}{2}\right) !\left(N_{f}-\frac{q}{2}\right) !} J^{2},
$$

which, in the limit $q \gg 1, N_{f} \gg 1$ such that $N_{f} \gg q$, naively, yields:

$$
J_{\text {eff }}^{2}=\frac{1}{N_{f}^{q}} \frac{1}{\left(\frac{q}{2}\right) !} J^{2} \rightarrow 0 .
$$

Thus, with a very large global symmetry, the emergent coupling is very weak. This implies that the resulting chaotic behaviour will be accompanied with a vanishingly small value of the Lyapunov exponent. Thus, we can tune the chaotic behaviour with a global flavour symmetry, as well. 


\section{Conclusion}

In this article, we have explored and demonstrated a tuneable Lyapunov exponent by introducing conserved charges in the system, even when the charge is a simple $\mathrm{U}(1)$. We have considered SYK-type models, with complex fermions and a $q$-body all-to-all randomized interaction, in the $q \rightarrow \infty$ limit. For these models, we have explicitly demonstrated that a non-vanishing chemical potential has an exponentially large dominance over the $q$-body interaction coupling strength, in determining the chaos behaviour. It is expected, from the structure of the Schwinger-Dyson equations, that similar features hold for the tensor models [19], which share many interesting properties of the SYK-type interaction, but without the disorder averaging.

There are various interesting directions for future explorations. Given the results above, one may explore higher dimensional generalizations of the SYK-model, e.g. the model in [24], with an introduction of conserved charges. One would, naívely, expect a similar behaviour of the resulting Lyapunov exponent for the higher dimensional models; however, it would be very interesting to check how the details fall into the right places. Staying within the theme of a tuneable chaos, motivated by the similarities of SYK-model behaviour and random matrix behaviour at late times, it is natural to incorporate the effect of conserved charges in random matrix theories and analyze the consequences at late times [35-37].

From a holographic perspective, our analysis suggests that by introducing bulk gauge fields that correspond to introducing chemical potentials for the dual boundary theory, one should be able to do away with chaos completely, or, at least, should be able to tune down the Lyapunov exponent from its' maximal value. This would be an interesting aspect to check explicitly. Towards that, one presumably begins with a gravity description in e.g. $(d+$ 1)-dimensional bulk with AdS-asymptotic, and studies a scattering problem, a la [38], in the presence of a global charge. On a similar note, it is also very intriguing to explore the possibility of constructing an SYK-type model from explicit D-brane construction in string theory, with or without global charges. One natural obstacle, for the SYK-type interaction, is to realize the dynamical origin of disorder averaging from the brane picture. Perhaps the large $N$ tensor models can emerge more naturally in such scenarios. We are currently exploring some of these issues further.

\section{Acknowledgments}

We thank Ashoke Sen and K. Sengupta for many interesting discussions. RB and SC would like to thank Saha Institute for hospitality and DPJ would like to thank IMSc for hospitality. Work of SC was partly supported by the Infosys scholarship for senior students.

Open Access. This article is distributed under the terms of the Creative Commons Attribution License (CC-BY 4.0), which permits any use, distribution and reproduction in any medium, provided the original author(s) and source are credited. 


\section{References}

[1] S. Strogatz, Nonlinear dynamics and chaos, Perseus Books, New York, U.S.A., (1994).

[2] A.I. Larkin and Yu.N. Ovchinnikov, Quasiclassical methods in the theory of superconductivity, JETP 28 (1969) 1200.

[3] S.H. Shenker and D. Stanford, Black holes and the butterfly effect, JHEP 03 (2014) 067 [arXiv: 1306.0622] [INSPIRE].

[4] S. Sachdev and J.-W. Ye, Gapless spin fluid ground state in a random, quantum Heisenberg magnet, Phys. Rev. Lett. 70 (1993) 3339 [cond-mat/9212030] [INSPIRE].

[5] A. Kitaev, A simple model of quantum holography (part 1), talk at KITP, http://online.kitp.ucsb.edu/online/entangled15/kitaev/, University of California, Santa Barbara CA U.S.A., 7 April 2015.

[6] A. Kitaev, A simple model of quantum holography (part 2), talk at KITP, http://online.kitp.ucsb.edu/online/entangled15/kitaev2/, University of California, Santa Barbara CA U.S.A., 27 May 2015.

[7] J. Maldacena and D. Stanford, Remarks on the Sachdev-Ye-Kitaev model, Phys. Rev. D 94 (2016) 106002 [arXiv:1604.07818] [INSPIRE].

[8] J. Maldacena, S.H. Shenker and D. Stanford, A bound on chaos, JHEP 08 (2016) 106 [arXiv: 1503.01409] [INSPIRE].

[9] J. Maldacena, D. Stanford and Z. Yang, Conformal symmetry and its breaking in two dimensional nearly anti-de-Sitter space, PTEP 2016 (2016) 12C104 [arXiv:1606.01857] [INSPIRE].

[10] S. Giombi, I.R. Klebanov and G. Tarnopolsky, Bosonic tensor models at large- $N$ and small $\epsilon$, arXiv: 1707.03866 [INSPIRE].

[11] I.R. Klebanov and G. Tarnopolsky, On large-N limit of symmetric traceless tensor models, JHEP 10 (2017) 037 [arXiv:1706.00839] [INSPIRE].

[12] D.J. Gross and V. Rosenhaus, A generalization of Sachdev-Ye-Kitaev, JHEP 02 (2017) 093 [arXiv: 1610.01569] [INSPIRE].

[13] V. Bonzom, L. Lionni and A. Tanasa, Diagrammatics of a colored SYK model and of an SYK-like tensor model, leading and next-to-leading orders, J. Math. Phys. 58 (2017) 052301 [arXiv: 1702.06944] [INSPIRE].

[14] Y. Gu, X.-L. Qi and D. Stanford, Local criticality, diffusion and chaos in generalized Sachdev-Ye-Kitaev models, JHEP 05 (2017) 125 [arXiv: 1609.07832] [INSPIRE].

[15] A.M. García-García, B. Loureiro, A. Romero-Bermúdez and M. Tezuka, Stability of chaos in a generalised Sachdev-Ye-Kitaev model, arXiv: 1707.02197 [INSPIRE].

[16] J. Polchinski and V. Rosenhaus, The spectrum in the Sachdev-Ye-Kitaev model, JHEP 04 (2016) 001 [arXiv: 1601.06768] [INSPIRE].

[17] I.R. Klebanov and G. Tarnopolsky, Uncolored random tensors, melon diagrams and the Sachdev-Ye-Kitaev models, Phys. Rev. D 95 (2017) 046004 [arXiv:1611.08915] [INSPIRE].

[18] W. Fu, D. Gaiotto, J. Maldacena and S. Sachdev, Supersymmetric Sachdev-Ye-Kitaev models, Phys. Rev. D 95 (2017) 026009 [Addendum ibid. D 95 (2017) 069904] [arXiv: 1610.08917] [INSPIRE]. 
[19] E. Witten, An SYK-like model without disorder, arXiv:1610.09758 [INSPIRE].

[20] T. Nishinaka and S. Terashima, A note on Sachdev-Ye-Kitaev like model without random coupling, arXiv:1611.10290 [INSPIRE].

[21] R. Gurau, The complete $1 / N$ expansion of a SYK-like tensor model, Nucl. Phys. B 916 (2017) 386 [arXiv: 1611.04032] [INSPIRE].

[22] C. Krishnan, S. Sanyal and P.N. Bala Subramanian, Quantum chaos and holographic tensor models, JHEP 03 (2017) 056 [arXiv:1612.06330] [INSPIRE].

[23] R. Gurau, The i€ prescription in the SYK model, arXiv:1705.08581 [INSPIRE].

[24] G. Turiaci and H. Verlinde, Towards a 2d QFT analog of the SYK model, JHEP 10 (2017) 167 [arXiv: 1701.00528] [INSPIRE].

[25] P. Narayan and J. Yoon, SYK-like tensor models on the lattice, JHEP 08 (2017) 083 [arXiv: 1705.01554] [INSPIRE].

[26] C. Krishnan and K.V.P. Kumar, Towards a finite-N hologram, JHEP 10 (2017) 099 [arXiv: 1706.05364] [INSPIRE].

[27] M. Berkooz, P. Narayan, M. Rozali and J. Simón, Higher dimensional generalizations of the SYK model, JHEP 01 (2017) 138 [arXiv:1610.02422] [INSPIRE].

[28] A. Jevicki, K. Suzuki and J. Yoon, Bi-local holography in the SYK model, JHEP 07 (2016) 007 [arXiv: 1603.06246] [INSPIRE].

[29] J. Murugan, D. Stanford and E. Witten, More on supersymmetric and $2 d$ analogs of the SYK model, JHEP 08 (2017) 146 [arXiv:1706.05362] [INSPIRE].

[30] J. Yoon, Supersymmetric SYK model: bi-local collective superfield/supermatrix formulation, JHEP 10 (2017) 172 [arXiv:1706.05914] [INSPIRE].

[31] D. Stanford and E. Witten, Fermionic localization of the Schwarzian theory, JHEP 10 (2017) 008 [arXiv: 1703.04612] [INSPIRE].

[32] R.A. Davison, W. Fu, A. Georges, Y. Gu, K. Jensen and S. Sachdev, Thermoelectric transport in disordered metals without quasiparticles: the Sachdev-Ye-Kitaev models and holography, Phys. Rev. B 95 (2017) 155131 [arXiv:1612.00849] [INSPIRE].

[33] K. Bulycheva, A note on the SYK model with complex fermions, arXiv:1706.07411 [INSPIRE].

[34] S. Banerjee and E. Altman, Solvable model for a dynamical quantum phase transition from fast to slow scrambling, Phys. Rev. B 95 (2017) 134302 [arXiv:1610.04619] [INSPIRE].

[35] A.M. García-García and J.J.M. Verbaarschot, Spectral and thermodynamic properties of the Sachdev-Ye-Kitaev model, Phys. Rev. D 94 (2016) 126010 [arXiv:1610.03816] [INSPIRE].

[36] J.S. Cotler et al., Black holes and random matrices, JHEP 05 (2017) 118 [arXiv: 1611.04650] [INSPIRE].

[37] A.M. García-García and J.J.M. Verbaarschot, Analytical spectral density of the Sachdev-Ye-Kitaev model at finite N, Phys. Rev. D 96 (2017) 066012 [arXiv:1701.06593] [INSPIRE].

[38] S.H. Shenker and D. Stanford, Stringy effects in scrambling, JHEP 05 (2015) 132 [arXiv: 1412.6087] [INSPIRE]. 